services, despite $80 \%$ having met a member of adult palliative care team. No patients had a formal transition plan. $50 \%$ of staff felt support for transition was inadequate, with $70 \%$ expressing the need for improved continuity in the service.

Conclusion A clear transition process is required to ensure early planning, that needs are met by adult services, and that family goals drive the transition process. Issues of identity, sexuality and spiritual development require specific focus. Funding, training and improved key working need to be addressed. A multidisciplinary approach with improved access to adult respite services is vital.

\section{P-218 MODEL OF CARE: A STRUCTURE AND PHILOSOPHY THAT EMPOWERS, ENABLES, EQUIPS ADULTS AND THEIR FAMILIES}

Ray Wilson, Mark Jarman- Howe, Laura Shukla. St Helena Hospice, Colchester, UK

\subsection{6/bmjspcare-2017-hospice.243}

Background Responding to stakeholder feedback and wider strategic developments, led us to review how we deliver care to be more social and personalised. Growing demand affected staff's ability to work in a structured way leading to unmet expectations.

Aims We adjusted our approach to multidisciplinary team assessments with the following aims for patients and families:

- To have an initial holistic assessment, using a SystmOne core template, by an appropriate hospice professional, not necessarily a nurse specialist

- Their goals are identified, with a view to achieving results within six sessions.

This new model's aims are to enable, equip and empower people by:

- Maximising their existing support network

- Sourcing alternative/additional support

- Providing hospice support (six sessions of care model) 'sticking like glue' to ensure issues are identified, goals set and a plan agreed.

Method A cross-organisation project group reviewed referral criteria and assessment processes. SystmOne templates were revised and the Outcome Assessment and Complexity Collaborative, Adult Social Care Outcomes Framework and Essex County Council's '3-conversation model' embedded in a core assessment. Staff were reorganised to enable a more responsive service.

Results Early indications are that this will achieve:

- Common understanding for hospice staff by working within one structure

- More comprehensive, timely crisis response - 'right person, right skills, right place, right time'

- Multi-disciplinary teams using their skills from first holistic assessment - 'right person, right skills, right place, right time'

- Widening access to the hospice

- Integrated health and social care assessment, adhering to Care Act 2014

- Measurable outcomes

- Model potentially to be replicated across the local health system

- All stakeholders clearer about what we offer.
Conclusion This model of care provides a clear structure for hospice professionals, empowers patients and families, ensures greater access to hospice care, responds to patient and family needs in a person-centred and timely way and provides evidence of outcomes and impact.

\section{P-219 THE EARLIER THE BETTER: EARLY REFERRAL TO PALLIATIVE CARE IMPROVES QUALITY OF LIFE}

Sue Eskins, Becky Avery, Lynn Porch, Sarah Parkes. Dorothy House Hospice Care, Wiltshire, UK

\subsection{6/bmjspcare-2017-hospice.244}

Background In 2014 the WHO declared that 'early palliative care not only improves quality of life for patients but also reduces unnecessary hospitalisation and use of health care services'. Following an audit in 2014 the Contact Centre at our hospice were aware that there were a number of patients who would benefit from our services but were either declining the services or being declined due to not being able to meet the criteria for specialist palliative care and end of life care.

Aims To develop an early palliative care service which allowed the patients and families/carers to experience a modern hospice approach of living well and palliative reablement. This included:

- Advance care planning

- Early support for families and carers

- Networking with other agencies e.g., CAB, carers support

- Live well approach

- Opportunity for referral to hospice services to prevent crisis e.g., 24 hour advice line, $\mathrm{H} @ \mathrm{H}$

- Psychological support.

Methods

- Outpatient style appointment at an Outreach Centre close to home at the patient's/family convenience with a named nurse.

- Patient centred approach

- Referral to other internal and services

- Flexible approach to use of hospice services.

Results

- Significant increase in the number of patients/families gaining access to hospice services

- Extension of lighter touch services

- Increase in referrals of non-cancer and long term conditions

- Positive feedback from patients/families

- Earlier referral for some patients to complex specialist services

- Development of the nursing team's skill mix

- De-stigmatises hospice care.

Conclusion

- Further investment in the early referral services as patients and families/carers are utilising living well and palliative reablement services

- An opportunity to encourage end of life conversations and advance care planning for patients with dementia

- Potential to extend into GP surgeries/satellite clinics to reduce anxiety around being referred to a hospice

- Potential to extend into outpatient services at local hospitals to support patients who are newly diagnosed as palliative. 


\section{P-220 MONEY, MONEY, MONEY: SUPPORTING THE FINANCIAL} WELFARE OF HOSPICE PATIENTS

Sandra Cayzer. Heart of Kent Hospice, Maidstone, UK

\subsection{6/bmjspcare-2017-hospice.245}

Background Reports show that finances are one of the top concerns for patients, their families and carers. On average a person is $£ 570$ per month worse off when diagnosed with cancer, with almost half of them not receiving any advice regarding their financial concerns from their healthcare professional.

Aim To establish a dedicated hospice welfare service. The specialist service to include, (but not exclusive to), advocacy and advice on welfare benefits, grants, tax, insurances, pensions, wills, power of attorney, probate, funeral costs, council tax, housing, utility bills, immigration, employment rights, carers rights and nursing home fees.

Method Recruitment of an experienced specialist adviser helped to establish the welfare service. Where other advice services are age, location or illness specific, hospice patients now receive tailored advice and support on a range of welfare/financial issues from hospice referral to bereavement and beyond.

Engagement with the welfare service enables patients to:

- Afford additional costs associated with illness

- Lessens the impact from loss of earnings through unemployment or reduced hours

- Reduce the need to draw on savings

- Plan finances for those left behind

- Positively impacts on wellbeing of patient, families and carers

- Carers able to concentrate on their primary function

- Bereaved able to address financial and legal issues, and ready to move onto counselling.

Conclusion The provision of a hospice welfare service complements the healthcare patients receive and enables healthcare staff to concentrate on the patient's medical needs. By addressing patient, family and carer financial concerns the welfare service enables patients to concentrate on their health and for families to have quality time with loved ones rather than worry about financial matters. It has the potential to increase the possibility that patients, family and carers will give greater consideration to donating to the hospice rather than other well- known national charities.

\section{P-221 COACHING FROM THE SIDE-LINES: CO-DEVELOPING A 'COMPASSIONATE COMMUNITY' SUPPORT GROUP}

Wayne de Leeuw, Lynn Porch. Dorothy House Hospice Care, Wiltshire, UK

\subsection{6/bmjspcare-2017-hospice.246}

Background Adapting the principle that 'care for one another at times of crisis and loss is not simply a task solely for health and social services but is everyone's responsibility' (Kellehear, 2015) and a need to find cost effective methods of delivering more support to more people; an innovative model of 'drop in support' was developed.

Aims of the Model A 'light touch', coaching style of group facilitation has been developed to enable anyone affected by the diagnosis of a life-limiting illness to develop coping strategies and in turn support others
To provide a safe setting for anyone affected by the diagnosis of a life-limiting illness to meet weekly in a supported and facilitated environment

Attendance will be open to any individual who is affected by a life-limiting illness, be they a 'patient', 'carer' or someone bereaved.

Results People with lived experience of illness, caring and grieving are supporting one another with compassion, requiring little 'professional' intervention. The cost to facilitate these sessions to such large numbers is a fraction of those usually associated with a traditional hospice day care. Attendance every week is in excess of over 40 people and three new groups have been set up in two new centres to meet increasing demand. Outside of the 'group' setting, self-sustaining local support networks have been established and shared, decreasing reliance on traditional public services. The model of coaching, empowerment and self-sustainability can be demonstrated by the addition of a new volunteer facilitator who originally joined the group two years ago as a bereaved widow.

Conclusion By creating a safe space and nurturing a compassionate community approach, people are creating their own networks of support and in turn decreasing their reliance on the health and social care system.

\section{P-222 INTEGRATED TAMESIDE EMERGENCY DEPARTMENT AND WILLOW WOOD START PROJECT: TRANSFORMING SPECIALIST CARE}

Nicola Byrne. Willow Wood Hospice, Ashton Under Lyne, UK

\subsection{6/bmjspcare-2017-hospice.247}

Background Our Integrated Care Organisation acknowledges that there are patients with life-limiting illnesses who attend the Emergency Department (ED) requiring symptom control without requiring admission to hospital. Our hospice day services have undergone modernisation to provide flexible specialist palliative care. The START Clinic at the hospice can support a timely discharge for those patients wishing to not be admitted to hospital who can be supported in the community. Patients with long term or potential life-limiting illness, including patients with dementia, can be referred.

Aims To provide rapid access to specialist palliative care including medical assessment

To support the patient and family to understand their disease progression/symptom management in order to plan their own care and treatment and potentially reduce the incidence of crisis management and unnecessary re-attendance to ED

To provide support to patients living with dementia and their families in a home or care home setting

To reduce the need for crisis interventions

To reduce the need for unnecessary re-attendance and potential admission to a busy acute hospital environment.

Methods The patient is deemed medically stable and does not require acute inpatient treatment for their condition. Integrated. Assessing clinician in ED rings through referral to START clinic. START clinic contacts patient at earliest opportunity or confirms patient is coming to START from ED if appropriate. Patient assessed in START by appropriate clinicians and treatment plan agreed with patient and carer. Links to community agencies made including telemedicine, social care etc. 